\title{
Periplaneta americana extract promotes intestinal mucosa repair of ulcerative colitis in rat $^{1}$
}

\author{
Nan-nan Xue ${ }^{\prime \# ~(D), ~ M i a o ~ H e " ~(D) ~, ~ Y u e ~ L i " \# ~(D) ~, ~ J u n-z h u ~ W u ' ~(D) ~, ~ W e n-w e n ~ D u l l ~(D) ~, ~ X i u-m e i ~ W u ' ~(D) ~, ~}$ \\ Zi-zhong Yang ${ }^{\mathrm{V}}$ (D) , Cheng-gui ZhangIV (D) , Qi-yan Liv (D) , Huai Xiao" (D) \\ ' Master, Yunnan Provincial, Key Laboratory of Entomological Biopharmaceutical R\&D, Dali University, Yunnan, \\ China. Acquisition of data, manuscript preparation. \\ " PhD, National-Local Joint Engineering Research Center of Entomoceutics, Dali University, Yunnan, China. \\ Critical revision, final approval. \\ II'Master, The People's Hospital of Linqing, Shandong Province, China. Acquisition, analysis and interpretation \\ of data. \\ IVPhD, Yunnan Provincial, Key Laboratory of Entomological Biopharmaceutical R\&D, Dali University, Yunnan, \\ China. Conception and design of the study, manuscript writing, final approval. \\ $\checkmark$ PhD, The First People's Hospital of Yunnan Province, Kunming, Yunnan Province, China. Histopathological \\ examinations, critical revision, final approval. \\ \# The authors contributed equally to this work
}

\begin{abstract}
Purpose: To investigate the mechanism of Periplaneta americana extract promoting intestinal mucosal repair of OXZ-induced colitis in rat.

Methods: All experiments used an equal number of male and female SD rats $(n=48)$. We injected OXZ into the colon to induce UC rat model. To determine the optimal concentration of $P$. Americana's extract (PA-40), it was classified into low (L), medium (M), and high $(H)$ doses. After OXZ treatment, each drug was administered by enema for 7 consecutive days. Rats were divided into the following 6 groups: (1) Saline treatment group (NC), (2) OXZ treatment UC model group (MC), (3) OXZ + budesonide group (BUN), (4) OXZ + PA-40 L group, (5) OXZ + PA-40 M group, (6) OXZ + PA-40 H group. Disease activity index (DAI) scores, colon length, histopathological score, serum cytokine level (IL-4, IL-10, iNOS, tNOS), and amount of MPO, EGF, IL-13 in colonic mucosa were measured.
\end{abstract}

Results: PA treatment had a significant healing effect on the OXZ-colitis model and significantly reduced the lesioned area, especially in the $\mathrm{PA}-40 \mathrm{H}$ groups. PA treatment did not alter the expression of IL-10 and MPO level, but increased EGF (epidermal growth factor) and decrease IL-13 in the colonic tissue. PA inhibited the rise of NOSs (nitric oxide synthase) and decreased the serum IL-4 level.

Conclusions: The data suggest that Periplaneta americana extract may be a potential compound for the treatment of colonic lesions. The mechanism may be related to inhibiting the secretion of IL-13 and promoting the formation of EGF.

Key words: Periplaneta. Oxazolone. Colitis, Ulcerative. Interleukin-13. Epidermal Growth Factor. Rats. 


\section{- Introduction}

Inflammatory bowel disease (IBD) is a group of inflammatory diseases that affect the gastrointestinal tract, including Crohn's disease (CD) and ulcerative colitis $(U C)^{1}$. UC is characterized by continuous inflammation of the lamina propria of the colon, accompanied by mucosal barrier damage and infiltration of inflammatory factors ${ }^{2}$. Clinical symptoms are abdominal pain, diarrhea, weight loss, mucopurulent blood stools and some common complications such as colon cancer ${ }^{3}$. It is the most common form of inflammatory bowel disease worldwide ${ }^{4}$. Although there has been significant progress in understanding the pathogenesis of IBD in the past few years, the main cause of IBD remains unclear. Environmental factors, immune factors and genetic susceptibility seem to promote the development of intestinal inflammation to a certain extent ${ }^{5-9}$. There are no treatments for reducing inflammation and restoring intestinal barrier function ${ }^{10}$. Commonly used clinical drugs for the treatment of UC include 5-aminosalicylic acid, corticosteroids, thiopurines, calcineurin inhibitors, anti-tumor necrosis factor (TNF) agents, antiadhesion molecules, JAK inhibitor ${ }^{11-13}$ and other drugs ${ }^{14}$. However, most drugs have some side effects or limited therapeutic effects. Oxazolone (OXZ), as a hapten, has shown to induce contact allergic reactions in all parts of the animal body. Studies at home and abroad have shown that its induced colitis is similar to human UC in histological features and inflammatory distribution ${ }^{15,16}$.

Insects are the world's largest animal population with over 700.000 species $^{17}$. More than 1,900 edible insects have been identified worldwide, including crickets, locusts, mealy larvae, ants, grasshoppers and others, which contain high-quality proteins, minerals, fatty acids, vitamins and amino acids for human consumption ${ }^{18,19}$. The consumption of insects may have positive effects on human health. For example, cricket powder may stimulate the growth of intestinal microbiota, reduce the level of TNF- $\alpha$ in plasma, and it is rich in chitin and chitosan, which can inhibit pathogenic microorganisms in the intestines ${ }^{20,21}$. Locust powder can inhibit intestinal lipid absorption by changing the content of short-chain fatty acids in rat cecum ${ }^{17}$. As a kind of medicinal and edible insect, Periplaneta americana has been recorded in many important classical documents of traditional Chinese medicine (TCM) such as "shen nong Ben cao jing"22. Modern medical research has found that Periplaneta americana has anti-tumor ${ }^{23}$, enhanced immunity, antibacterial, anti-inflammatory and analgesic effects, tissue repair and so on ${ }^{24}$. Our previous study found that it has a good therapeutic effect on ulcerative colitis, but its mechanism of action is still unclear. As an extract of Periplaneta americana, PA-40 has been widely used in the treatment of ulcerative colitis in China, and has achieved good results. In this study, the therapeutic effect of PA-40 on OXZ-induced ulcerative colitis in rats was investigated, and the mechanism of action was preliminarily explored.

\section{- Methods}

\section{Reagents}

Oxazolone (4-ethoxymethyl-2-phenyl-2-oxazolinone-5-1) was purchased from China shenzhen regent biochemical technology CO. LTD (Shenzhen, China). The occult blood kit, MPO (myeloperoxidase), NOS test kits and rat interleukin-13 ELISA kit were purchased from jiangsu nanjing jiancheng biotechnology CO. LTD (Nanjing, China). Rat interleukin-4 (IL-4), interleukin-10 (IL-10) and epidermal growth factor (EGF) ELISA kits were purchased from xinbosheng biotechnology CO. LTD (Shenzhen, China).

\section{Preparation of PA-40 in Periplaneta americana extract}

Periplaneta americana was dried, crushed, sifted with 20 mesh, soaked in $80 \%$ ethanol solution of 10 times the amount for $2 \mathrm{~h}$, then heated at $70^{\circ} \mathrm{C}$ for 4 $h$, extracted twice, combined with filtrate, and then evaporated for concentration. After D101 macroporous resin was applied, the column volume was eluted with pure water and then eluted with $40 \%$ ethanol. The $40 \%$ ethanol elution part was collected and concentrated into a viscous extract to obtain Periplaneta americana extract PA-40.

\section{Experimental animals}

Sprague-Dawley rats (180-220g, equal ration of male and female) were obtained from Hunan SJA Laboratory Animal CO. LTD .The experiments follow the rules of the school's animal ethics committee, which approves all experiments for the purpose of controlling and supervising animal experiments (protocol number 2015-0820).

\section{Establishment of UC model}

OXZ induced colitis method has been described by Heller etc ${ }^{25}$; the back skin of rats is shaved $(2 \mathrm{~cm} \times 2 \mathrm{~cm})$, and shaving drops of $0.2 \mathrm{~mL} 3.0 \% \mathrm{OXZ}$ solution (dissolved in anhydrous ethanol) are used for seven days; after 7 
days, a suitable amount of isoflurane anesthesia is used with each rat, and then a $2 \mathrm{~mm}$ diameter polyethylene tube is inserted into the large intestine about $8 \mathrm{~cm}$, with $1.0 \%$ of OXZ solution (dissolved in $50 \%$ ethanol) 1.0 $\mathrm{mL} \cdot \mathrm{kg}^{-1}$. The inversion of rats is performed $1 \mathrm{~min}$ after injection to prevent backflow.

\section{Experimental grouping}

Normal control group (NC) was given normal saline (Enema, $2.5 \mathrm{ml} \cdot \mathrm{kg}^{-1}$ ), and the model rats were stratified with DAl score according to Hamamoto etc ${ }^{26}$. After removing the rats with very mild inflammatory model, they were randomly divided into model group (MC), BUN group, and three groups with low, medium and high doses of PA-40, with 8 rats in each group. Rats in the MC group were given normal saline (Enema, $2.5 \mathrm{~mL} \cdot \mathrm{kg}^{-1}$ ) and rats in the budesonide group (BUN) (Enema, $0.2 \mathrm{mg} \cdot \mathrm{kg}^{-1}$ ) were given three different doses of PA-40 (Enema, 50, 100 and $200 \mathrm{mg} \cdot \mathrm{kg}^{-1}$, labeled as PA-40 L, PA-40 M, PA$40 \mathrm{H}$ ). Daily weight, fecal viscosity, and blood stools of all rats were recorded for 7 days after administration, and OXZ-induced colitis was assessed using the disease activity index (DAI) scoring system.

\section{The disease activity index}

The daily clinical assessment of the rat after the challenge included measurement of body weight and evaluation of stool consistency and the presence of blood in the stools by occult blood kit, which was graded according to Table 1.

Table 1 - DAl scoring system.

\begin{tabular}{|c|c|c|c|}
\hline Score & Weight loss(\%) & Stool consistency & Hematochezia \\
\hline 0 & 0 & Normal & Normal \\
\hline 1 & $\geq 1-<5$ & Semi loose $(+)$ & Feces with Occult blood (+) \\
\hline 2 & $\geq 5-<10$ & Semi loose $(++)$ & Feces with Occult blood $(++)$ \\
\hline 3 & $\geq 10-<15$ & Loose $(+)$ & Bloody feces $(+)$ \\
\hline 4 & $\geq 15$ & Loose $(++)$ & Bloody feces $(++)$ \\
\hline
\end{tabular}

\section{Macroscopical evaluation of colitis colon}

All rat colon tissues were rapidly stripped and their natural extension length was measured. After the colon was removed, the intestinal contents were flushed with sterile saline. The water was dried with a sterile blotting paper and the wet weight of the colon was measured. The colon mucosal damage index (CMDI) was graded according to Table 2.

Table 2 - CMDI rating criteria.

\begin{tabular}{|c|c|}
\hline Score & CMDI scoring criteria \\
\hline 0 & no damage \\
\hline 1 & mild hyperemia edema, smooth surface, no erosion or ulcer \\
\hline 2 & Hyperemia and edema, rough mucosa, granular, erosion or intestinal adhesion \\
\hline 3 & $\begin{array}{r}\text { High hyperemia and edema, necrosis and ulceration on the surface, area }<1 \mathrm{~cm}^{2} \text {, intestinal wall thickening or } \\
\text { necrosis and inflammatory polyps on the surface }\end{array}$ \\
\hline 4 & $\begin{array}{c}\text { Severe congestive edema, mucosal necrosis and ulceration, } \geq 1 \mathrm{~cm}^{2} \text { or necrosis of the entire intestinal wall, } \\
\text { toxic megacolon leading to death }\end{array}$ \\
\hline
\end{tabular}

\section{Histopathological evaluation}

After the colon was macroscopical evaluated, it was fixed with $10 \%$ buffered formaldehyde, embedded in paraffin, sectionalized, and stained with hematoxylin and eosin (HE). Sections of colon tissue were analyzed under a microscope and photographed for preservation. The histopathological score was graded according to Table 3 . 
Table 3 - Histopathological score.

\begin{tabular}{lcc}
\hline Score & Epithelial cell & Inflammatory cell infiltration \\
\hline 0 & Normal & no infiltration \\
1 & Cup cell loss & Infiltrate into the basal layer of the crypt \\
2 & Large areas of goblet cell loss & Infiltrates into the muscularis mucosa \\
3 & Loss of crypt cells & Infiltration extends deep into the muscularis mucosa, accompanied by \\
4 & Extensive loss of crypt cells & Infiltration reaches the submucosa
\end{tabular}

\section{Measurement of IL-13, MPO and EGF levels in colonic tissue}

Colonic tissue was suspended in ice-cold PBS and then simply homogenized. The homogenate was centrifuged at $12000 \mathrm{rpm}, 4^{\circ} \mathrm{C}$ for $10 \mathrm{~min}$, and the supernatant was collected. The contents of IL-13 and epidermal growth factor (EGF) were determined by enzyme-linked immunosorbent assay. The content of MPO in colon tissues was determined as per kit requirements.

\section{Determination of IL-4, IL-10, iNOS and tNOS in blood serums}

The whole blood samples of all rats were collected. They were centrifuged immediately at $12000 \mathrm{~g}, 4{ }^{\circ} \mathrm{C}$ for $10 \mathrm{~min}$; then, the supernatant was collected and measured at $-20{ }^{\circ} \mathrm{C}$. The contents of IL- 4 and IL-10 were determined by elisa kit, and the operation was carried out according to the kit instruction's requirements. Nitric oxide synthase (NOS) in the serum was measured according to the kit instructions.

\section{Statistical analysis}

All experiment results were expressed as mean \pm standard deviation (SD). One-way analysis of variance (ANOVA) and post-hoc analyses were used for the analysis of differences between groups. The SPSS 20.0 and GraphPad Prism software were used to generate a $P$-value and statistic for each analysis; P-value less than 0.05 was considered statistically significant.

\section{- Results}

\section{Ingredients of $P A-40$}

The polypeptide content was carried out by Lowry method using bovine serum albumin as a standard. The PA-40 polypeptide content was $51.54 \%(\mathrm{~g} / \mathrm{g})$ by the Lowry method. After the HPLC analysis of the chemical composition of PA-40, 5 compounds were identified in PA-40. They were uracil, hypoxanthine, uridine, adenosine and inosine (Fig. 1).

\section{Disease activity index (DAI)}

A combinatorial DAl, considering percentage body weight loss, stool consistency, and bloody stool was used to evaluate the activity of the disease and the response to treatment. After 7 days of continuous enema, different groups of rats showed different degrees of enteritis symptoms. Compared with the NC group, the DAI score of rats in the $M C$ group increased significantly. PA-40 L, $\mathrm{PA}-40 \mathrm{M}$ and $\mathrm{PA}-40 \mathrm{H}$ groups reduced DAI scores. But the treatment effect of the PA-40 H group $\left(200 \mathrm{mg} \cdot \mathrm{kg}^{-1}\right)$ was better than that of the two other dosages (Fig. 1C).

\section{Macroscopical evaluation of colitis colon}

As shown in Figure $1 \mathrm{~A}, \mathrm{OXZ}$ enema induced gross colonic mucosal injury, such as hyperaemia, oedema, wall thickness, necrosis, and ulceration compared to the control group that showed no mucosal damage (MC). These observations were similar to pathological changes in human IBD. Enema with BUN and PA-40 can significantly improve the symptoms of colonic mucosal injury (Fig. 1D). The length of the colon was significantly shortened by $O X Z$, and the length of the colon in the model group $(15.63 \pm 1.77 \mathrm{~cm})$ was significantly shorter than that in the NC group $(20.04 \pm 1.22 \mathrm{~cm})$ (Fig. $1 \mathrm{~A}, \mathrm{E})$. Compared with the model group, the colon length of rats in the PA-40 treatment group was increased. But only the $\mathrm{PA}-40 \mathrm{H}$ group $(17.63 \pm 1.74 \mathrm{~cm})$ showed significant differences from the model.

\section{Histopathological evaluation}

The NC group had complete crypt structure and epithelial cell layer, and the goblet cell morphology was normal. In the MC group, the lamina propria was damaged, epithelial cells were lost, inflammatory cells were strongly infiltrated, large areas of intestinal crypts 
were necrotic, bleeding and edema were observed, and neutrophils were severely infiltrated and penetrated to the muscle layer. The mucosa and crypt regenerated in BUN group, and the inflammatory response was decreased. In the PA-40 L group, the lamina propria recess disappeared, with superficial mucosal ulcer and inflammatory cell infiltration. PA-40 M group showed crypt dilatation, deformation and lamina propria fibrosis. PA-40 $\mathrm{H}$ group had normal recess structure and no inflammatory characteristics (Fig. 1B).
A

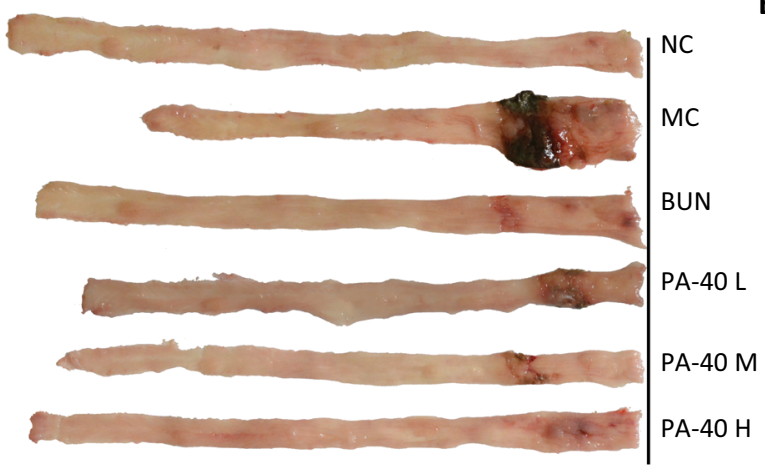

C



B

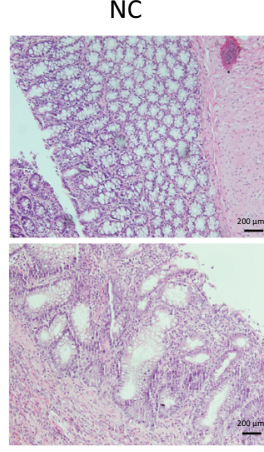

PA-40 L

D

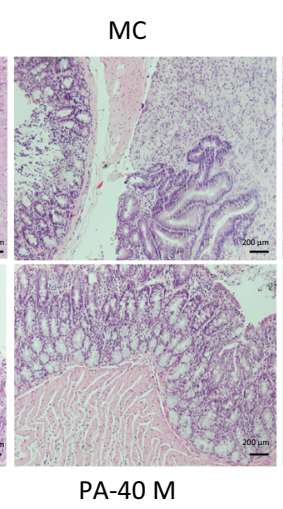

E
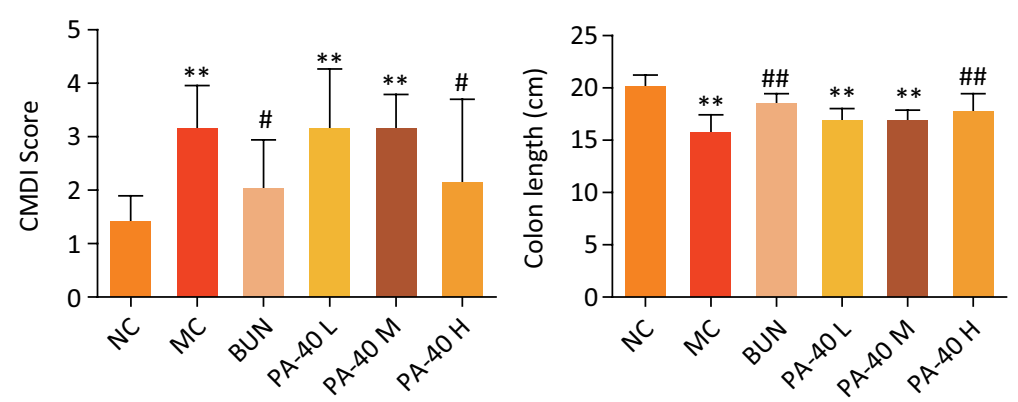

Figure 1 - Morphological analysis of OXZ-induced colitis rat treated with PA-40. (A) Samples of macroscopic findings of colonic mucosa. OXZ treatment group (MC) showed obvious bleeding, hyperemia and edema. (B) Representative histopathological images of rat colorectum (scale bar: $200 \mu \mathrm{m}$ ). MC group showed the loss of goblet cells, crypts and severe infiltration of inflammatory cells. (C) Time-dependent changes of DAI scores for 7 days by PA40 and BUN treatment. (D) The colon mucosal damage index (CDMI) of colon tissue samples. PA-40 $\mathrm{H}$ group significantly reduced the score compared with MC group. (E) The comparison of colon length between 6 groups. PA-40 treatment significantly ameliorated the colon length compared with $\mathrm{MC}$ group. Data are presented as mean \pm SD of 8 rats in each group (**, $\mathrm{P}<0.01$ vs. NC group; \#, $\mathrm{P}<0.05, \#, \mathrm{P}<0.01$ vs. MC group).

\section{Analysis of cytokine levels in colonic tissue}

The expression levels of IL-13 and MPO in the colonic tissues of $\mathrm{MC}$ rats were significantly up-regulated, compared with the NC group. After PA-40 treatment, the expression levels of IL-13 and MPO in colon tissues were significantly reduced $\left(* P<0.05,{ }^{*} P<0.01\right.$; $\# P<0.01$, Fig. 2A, B). It is suggested that PA-40 has certain anti-inflammatory effect on oxazolone-induced colitis. The colonic tissue EGF activity of rats in the MC group was significantly reduced, compared with the NC group. The activity of EGF in colon tissues of PA$40 \mathrm{M}$ and $\mathrm{PA}-40 \mathrm{H}$ group was significantly increased compared with that of $\mathrm{MC}$ group $\left({ }^{*} P<0.01 ;{ }^{\#} P<0.01\right.$, Fig. 2C). 
A



B

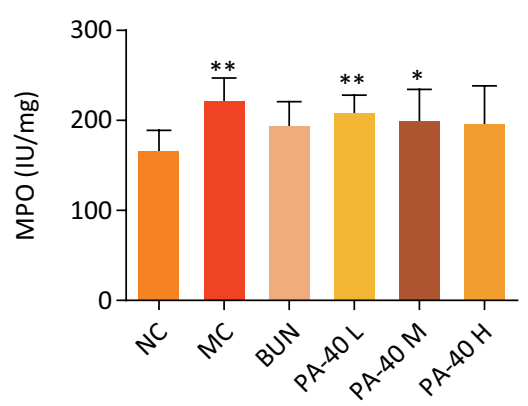

C



Figure 2 - Anti-inflammatory and cell proliferation effects of PA-40 in OXZ-induced colitis rat. The expression levels of IL-13 (A), EGF (C) were determined by ELISA kit. MPO (B) levels in colonic tissues were determined by MPO kit. Data are presented as mean \pm SD of 8 rats in each group $\left(*, P<0.05, * *, P<0.01\right.$ vs. NC group; ${ }^{*}, P<0.05,{ }^{*}, P<0.01$ vs. MC group).

\section{Analysis of cytokine levels in blood serums}

The expression level of IL-4 in the MC group significantly decreased, compared with the NC group, $(* * P<0.01$, Fig. $3 \mathrm{~A})$, and IL-10 decreased slightly $\left({ }^{*} P>0.05\right.$, Fig. $\left.3 \mathrm{~B}\right)$, but there was no statistical difference. The expression level of IL-4 in the PA-40 H group was significantly increased, compared with the
MC group ("\# $P<0.01$, Fig. 3A). The expression level of IL-10 was slightly increased (Fig. 3B). The expression levels of iNOS and tNOS in the MC group significantly increased, compared with the NC group ( ${ }^{* *} P<0.01$, Fig. 3C, D), and the expression levels of iNOS in the PA-40 $M$ and PA-40 $\mathrm{H}$ groups significantly decreased ("\# $P<0.01$, Fig. 3C).
A

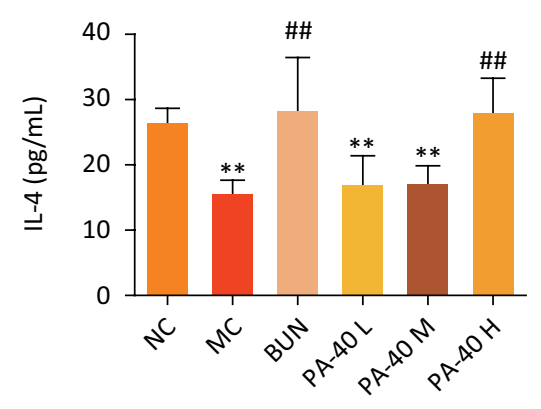

C

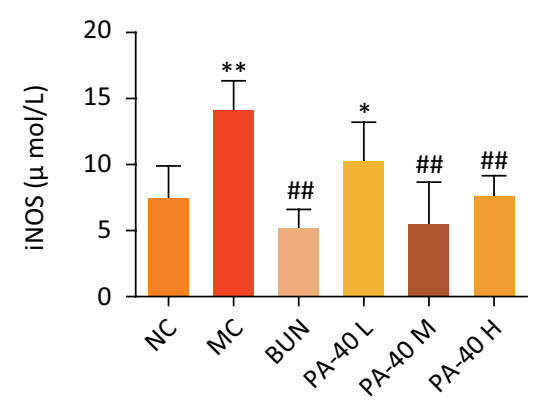

B



D



Figure 3 - Anti-inflammatory effects of PA-40 in OXZ-induced colitis rat. The expression levels of IL-4 (A), IL-10 (B), were determined by ELISA kit. iNOS (C), tNOS (D) levels in blood serums were determined by NOS kit. Data are presented as mean \pm SD of 8 rats in each group $\left(*, P<0.05, * *, P<0.01\right.$ vs. NC group; ${ }^{*}, \mathrm{P}<0.05,{ }^{*}, \mathrm{P}<0.01$ vs. MC group). 


\section{- Discussion}

At present, the methods for establishing animal models of IBD mainly include spontaneous animal model, genotype animal model, cell transplantation animal model and chemical drug induction animal model, among which chemical drug induction is the most extensive. In our previous experiment, Dinitrochlorobenzene (DNCB) combined with acetic acid was used to induce the rat colitis model. DNCB is a hapten chemical, which induces delayed intestinal mucosal response and imbalance of Th1/Th2 immune cells in rats after repeated sensitization ${ }^{27}$. Acetic acid can increase intestinal mucosal vascular permeability, activate kinin, interfere with blood coagulation, and initiate inflammation, thus forming local inflammatory lesions ${ }^{28}$. OXZ-induced colitis is a typical model of Th2 colitis associated with immunity ${ }^{29}$. Due to its simple and repeatable properties, the induced colonic intervention model is widely used to screen potential therapeutic drugs and the model is similar to human UC, which can be used for further study of the disease.

In OXZ colitis, IL-4 is the initial cytokine produced by oxazolone colitis, but IL- 4 is soon replaced by the production of IL-13, which can activate epithelial cells to secrete mucus and fluid ${ }^{25}$. This cytokine and IL-4 can lead to the destruction of tight connections between epithelial cells, which in turn opens the door to bacterial invasion. When IL-13 was neutralized, oxazolone administration did not cause any histological changes or other inflammatory symptoms ${ }^{30}$. This strongly suggests that IL-13 mediates mucosal tissue damage as an effector cytokine ${ }^{31}$. The expression level of IL-4 in serum of BUN and PA-40 group was significantly increased, and the level of IL-13 was significantly decreased. It is suggested that Periplaneta americana extract may promote the repair of intestinal mucosa by inhibiting the expression of IL-13 in UC rats ${ }^{32}$. IL-10, mainly secreted by Th 2 cells, is an anti-inflammatory immune factor with immunomodulatory and anti-inflammatory effects, and plays an important role in regulating intestinal immune balance ${ }^{33}$. IL-10 showed no significant changes after treatment of BUN and PA-40. This may be because of the different sampling sites, or BUN and PA-40 inhibited Th2 responses.

Intestinal mucosal barriers include mechanical barriers, chemical barriers, immune barriers and biological barriers ${ }^{34}$. Epidermal growth factor (EGF) is an important cell growth factor that plays an important role in maintaining the intestinal mucosal barrier ${ }^{35,36}$. Supported by EGF, gastrointestinal mucosa has a significant ability to repair damage, and EGF stimulates cell migration and extracellular matrix formation ${ }^{37}$. EGF promotes wound healing by improving the integrity of intestinal epithelial and mucosal barriers in rats. The results of this experiment showed that BUN, PA-40 $\mathrm{M}$ and $\mathrm{H}$ dose groups could significantly improve the expression level of EGF.

Myeloperoxidase is a $140-\mathrm{kDa}$ heme protein secreted primarily by neutrophils and stored in eosinophils ${ }^{38,39}$. Colonic injury leads to the activation of neutrophils and macrophages, and the release of MPO from activated neutrophils causes hydrogen peroxide $\left(\mathrm{H}_{2} \mathrm{O}_{2}\right)$ to produce hypochlorous acid $(\mathrm{HOCl})$ and chlorine anions $\left(\mathrm{Cl}^{-}\right)$to further damage the colon ${ }^{40}$. The activity of MPO is often used as an indirect indicator of tissue severity. In this study, BUN and PA- $40 \mathrm{H}$ group could reduce the activity of intestinal mucosal MPO and reduce inflammatory response. But there was no significant difference. Physiological dose of NO can protect the digestive system, but excessive NO can stimulate the digestive system and lead to UC $C^{41}$. NOS is a rate-limiting enzyme for the synthesis of NO, which can indirectly reflect the level of NO. iNOS continuously release a large amount of $\mathrm{NO}$ after a certain stimulation can aggravate the degree of inflammation of the colon, and the higher the content of iNOS, the more serious the inflammation of UC. The results of this study suggested that in the OXZ-colitis model group, iNOS expression and NO release were increased, and NO and iNOS were positively correlated with MPO activity. After drug treatment, BUN, PA-40 treatment group of NO, iNOS expression significantly decreased. These results further confirmed that the extract of Periplaneta americana PA-40 has a certain therapeutic effect on OXZ-induced colitis.

\section{- Conclusion}

This study suggested that the therapeutic effect of PA-40 on UC may be realized by regulating the expression of related immune regulatory factors, inhibiting the expression of pro-inflammatory cytokines, and participating in promoting the repair of intestinal mucosa.

\section{- References}

1. Maheshwari RA, Balaraman R, Sailor GU, Sen DB. Protective effect of simvastatin and rosuvastatin on trinitrobenzene sulfonic acid-induced colitis in rats. Indian J Pharmacol. 2015;47:17-21. doi: 10.4103/0253-7613.150311

2. Naganuma M, Sugimoto $S$, Mitsuyama K. Efficacy of Indigo Naturalis in a multicenter randomized controlled trial of patients with ulcerative colitis. Gastroenterology. 2018;154:935-47. doi: 10.1053/j.gastro.2017.11.024. 
3. Feuerstein JD, Cheifetz AS. Ulcerative colitis: epidemiology, diagnosis, and management. Mayo Clinic Proc. 2014;89:1553-63. doi: 10.1016/j.mayocp.2014.07.002.

4. Lynch WD, Hsu R. Ulcerative colitis. In: StatPearls. Treasure Island (FL): StatPearls Publishing; 2020.

5. Shanahan F. Inflammatory bowel disease: immunodiagnostics, immunotherapeutics, and ecotherapeutics. Gastroenterology. 2001;120:622-35. doi: 10.1053/gast.2001.22122.

6. Rutgeerts $P$, Sandborn WJ, Feagan BG, Reinisch W, Olson A, Johanns J, Travers S, Rachmilewitz D, Hanauer SB, Lichtenstein GR. Infliximab for induction and maintenance therapy for ulcerative colitis. N Engl J Med. 2005;353:246276. doi: 10.1056/NEJMoa050516.

7. Reinisch W, Sandborn WJ, Rutgeerts $P$, Feagan BG, Rachmilewitz D, Hanauer SB, Lichtenstein GR, de Villiers WJ, Blank $M$, Lang $Y$. Long-term infliximab maintenance therapy for ulcerative colitis: the ACT-1 and -2 extension studies. Inflamm Bowel Dis. 2012;18:201-11. doi: 10.1002/ ibd.21697.

8. Xavier RJ, Podolsky DK. Unravelling the pathogenesis of inflammatory bowel disease. Nature. 2007;448:427-34. doi: $10.1038 /$ nature06005.

9. Knights D, Lassen KG, Xavier RJ. Advances in inflammatory bowel disease pathogenesis: linking host genetics and the microbiome. Gut. 2013;62:1505-10. doi: 10.1136/ gutjnl-2012-303954.

10. Vargas-Robles H, Castro-Ochoa KF, Citalán-Madrid AF, Schnoor M. Beneficial effects of nutritional supplements on intestinal epithelial barrier functions in experimental colitis models in vivo. World J Gastroenterol. 2019;25(30):418198. doi: 10.3748/wjg.v25.i30.4181.

11. Cohen RD, Woseth DM, Thisted RA, Hanauer SB. A metaanalysis and overview of the literature on treatment options for left-sided ulcerative colitis and ulcerative proctitis. Am J Gastroenterol. 2000;95:1263-76. doi: 10.1111/j.1572-0241.2000.01940.x.

12. Campieri M. New steroids and new salicylates in inflammatory bowel disease: a critical appraisal. Gut. 2002;50 Suppl 3:iii43-6. doi: 10.1136/gut.50.suppl_3.iii43.

13. Huang YF, Zhou JT, Qu C, Dou YX, Huang QH, Lin ZX, Xian YF, Xie JH, Xie YL, Lai XP. Anti-inflammatory effects of Brucea javanica oil emulsion by suppressing NF-KB activation on dextran sulfate sodium-induced ulcerative colitis in mice. J Ethnopharmacol. 2017;198:389-98. doi: 10.1016/j. jep.2017.01.042.

14. Kokkinidis DG, Bosdelekidou EE, Iliopoulou SM. Emerging treatments for ulcerative colitis: a systematic review. Scand J Gastroenterol. 2017;52:923-31. doi: 10.1080/00365521.2017.1326163.

15. Weigmann B, Neurath MF. Oxazolone-induced colitis as a model of Th2 immune responses in the intestinal mucosa. Methods Mol Biol. 2016;1422:253-61. doi: 10.1007/978-14939-3603-8_23.

16. Zhang L, Cao N, Wang Y, Wang Y, Wu C, Cheng X, Wang C. Improvement of oxazolone-induced ulcerative colitis in rats using andrographolide. Molecules. 2019;25(1):76. doi: 10.3390/molecules25010076.
17. Ochiai $M$, Inada $M$, Horiguchi $S$. Nutritional and safety evaluation of locust (Caelifera) powder as a novel food material. J Food Sci. 2020;85(2):279-88. doi: 10.1111/17503841.15024.

18. Montowska M, Kowalczewski Pt, Rybicka I, Fornal E. Nutritional value, protein and peptide composition of edible cricket powders. Food Chem. 2019;289:130-8. doi: 10.1016/j.foodchem.2019.03.062.

19. van Huis A. Potential of insects as food and feed in assuring food security. Ann Rev Entomol. 2013;58:563-83. doi: 10.1146/annurev-ento-120811-153704.

20. Stull VJ, Finer E, Bergmans RS, Febvre HP, Longhurst C, Manter DK, Patz JA, Weir TL. Impact of edible cricket consumption on gut microbiota in healthy adults, a doubleblind, randomized crossover trial. Sci Rep. 2018;8(1):10762. doi: 10.1038/s41598-018-29032-2.

21. Liu P, Piao XS, Thacker PA, Zeng ZK, Li PF, Wang D, Kim SW. Chito-oligosaccharide reduces diarrhea incidence and attenuates the immune response of weaned pigs challenged with Escherichia coli K88. J Anim Sci. 2010;88(12):3871-9. doi: 10.2527/jas.2009-2771.

22. Li LJ, Wang MZ, Yuan TJ, Xu XH, Dad HA, Yu CL, Hou J, Peng LH. The crude ethanol extract of Periplaneta americana L. stimulates wound healing in vitro \& in vivo. Chin Med. 2019;14:33. doi: 10.1186/s13020-019-0259-4.

23. Zhao Y, Yang A, Tu P, Hu Z. Anti-tumor effects of the American cockroach, Periplaneta americana. Chin Med. 2017;12:26-31. doi: 10.1186/s13020-017-0149-6.

24. Nguyen T, Chen X, Chai J, Li R, Han X, Chen X, Liu S, Chen $M, X u X$. Antipyretic, anti-inflammatory and analgesic activities of Periplaneta americana extract and underlying mechanisms. Biomed Pharmacother. 2020;123:109753-63. doi: 10.1016/j.biopha.2019.109753.

25. Heller F, Fuss IJ, Nieuwenhuis EE, Blumberg RS, Strober W. Oxazolone colitis, a Th2 colitis model resembling ulcerative colitis, is mediated by IL-13-producing NK-T cells. Immunity. 2002;17:629-38. doi: 10.1016/s1074-7613(02)00453-3.

26. Hamamoto N, Maemura K, Hirata I, Murano M, Sasaki $S$, Katsu K. Inhibition of dextran sulphate sodium (DSS)induced colitis in mice by intracolonically administered antibodies against adhesion molecules (endothelial leucocyte adhesion molecule-1 (ELAM-1) or intercellular adhesion molecule-1 (ICAM-1)). Clin Exp Immunol. 1999;117:462-8. doi: 10.1046/j.1365-2249.1999.00985.x.

27. Yuqing J, Qiang J, Xuezheng Z, Tao M, Yuanyuan L, Hongli L, Yufei $D$. Study on proliferation and activation of lymphocytes induced by 2, 4-dinitrochlorobenzene in hypersensitive dermatitis mice. China Occup Med. 2014;41(5):489-95.

28. Shengshuai L, Pengchuan W, Yusheng $X$, Changbao Y,Tian H,Yongmei S, Miao S, Ping W, Xiumei W, Protective effect of Periplaneta Americana extract in ulcerative colitis rats induced by dinitrochlorobenzene and acetic acid. Pharm Biol. 2016;54:2560-7.

29. Shih DQ, Targan SR. Immunopathogenesis of inflammatory bowel disease. World J Gastroenterol. 2008;14:390-400. doi: 10.3748/wjg.14.390.

30. Fuss IJ, Strober W. The role of IL-13 and NK T cells in experimental and human ulcerative colitis. Mucosal Immunol. 2008;1 Suppl 1:31-3. doi: 10.1038/mi.2008.40. 
31. Hoving JC. Targeting IL-13 as a host-directed therapy against ulcerative colitis. Front Cell Infect Microbiol. 2018;8:395. doi: 10.3389/fcimb.2018.00395.

32. Wang X, Fan F, Cao Q. Modified Pulsatilla decoction attenuates oxazolone-induced colitis in mice through suppression of inflammation and epithelial barrier disruption. Mol Med Rep. 2016;14(2):1173-9. doi: 10.3892/mmr.2016.5358.

33. Wang G, Xu B, Shi F, Du M, Li Y, Yu T, Chen L. Protective effect of methane-rich saline on acetic acid-induced ulcerative colitis via blocking the TLR4/NF-kappaB/MAPK pathway and promoting IL-10/JAK1/STAT3-mediated anti-inflammatory response. Oxid Med Cell Longev. 2019;2019:1-12. doi: 10.1155/2019/7850324.

34. Gai W, Na W, Jingxia $\mathrm{H}$. Study on the protective effect of curcumin on intestinal epithelial cell barrier damage. Gastroenterology. 2016;21(1):26-9.

35. Lawrence JP, Brevetti L, Obiso RJ. Effects of epidermal growth factor and Clostridium difficile toxin $B$ in a model of mucosal injury. J Pediatr Surg. 1997;32:430-3. doi: 10.1016/s0022-3468(97)90598-4.

36. van Landeghem L, Chevalier J, Mahé MM. Enteric glia promote intestinal mucosal healing via activation of focal adhesion kinase and release of proEGF. Am J Physiol Gastrointest Liver Physiol. 2011;300:G976-87. doi: 10.1152/ajpgi.00427.2010.
37. Konturek SJ, Brzozowski T, Majka J, Dembinski A, Slomiany A, Slomiany BL. Transforming growth factor alpha and epidermal growth factor in protection and healing of gastric mucosal injury. Scand J Gastroenterol. 1992;27:649-55. doi: 10.3109/00365529209000134.

38. Triantafyllidis I, Poutahidis T, Taitzoglou I, Kesisoglou I, Lazaridis C, Botsios D. Treatment with Mesna and n-3 polyunsaturated fatty acids ameliorates experimental ulcerative colitis in rats. Int J Exp Pathol. 2015;96:433-43. doi: 10.1111/iep.12163.

39. Kannan N, Guruvayoorappan C. Protective effect of Bauhinia tomentosa on acetic acid induced ulcerative colitis by regulating antioxidant and inflammatory mediators. Int Immunopharmacol. 2013;16:57-66. doi: 10.1016/j.intimp.2013.03.008.

40. Vinod P, Guruvayoorappan C. Protective effect of marine mangrove Rhizophora apiculata on acetic acid induced experimental colitis by regulating anti-oxidant enzymes, inflammatory mediators and nuclear factor-kappa B subunits. Int Immunopharmacol. 2014;18:124-34. doi: 10.1016/j.intimp.2013.11.007.

41. Guslandi M. Nitric oxide and inflammatory bowel diseases. Eur J Clin Invest. 1998;28:904-7. doi: 10.1046/j.13652362.1998.00377.x.

\section{Correspondence:}

Huai Xiao

Yunnan Provincial Key Laboratory of Entomological Biopharmaceutical R\&D

Dali, Yunnan China

Phone: 0872-2214251

422022546@qq.com
Conflict of interest: none

Financial sources: The National Natural Science Fund of China (NO.81660186, 81660605, 81860742, 81860765); Yunnan Fundamental Research Projects (grant NO. 2017FH001-108, 2018FB143, 2019FH001-003, 202001AT070021); Innovation Team of Dali University for Advanced Pharmaceutics of Entomological Biopharmacy R\&D (NO.ZKLX2019101); Innovative Team of Dali University for Digital Development of Medicinal Insects and Arachnids Resources (NO.ZKLX2020303); Special Fund for Traditional Chinese Medicine Pieces of Department of Industry and Information Technology of Yunnan Province (NO.2019-YG-067)

${ }^{1}$ Research performed at Yunnan Provincial Key Laboratory of Entomological Biopharmaceutical R\&D, School of Pharmacy and Chemistry, Dali University, Yunnan, China.

Received: June 06, 2020

Review: Aug 03, 2020

Accepted: Sept 04, 2020 